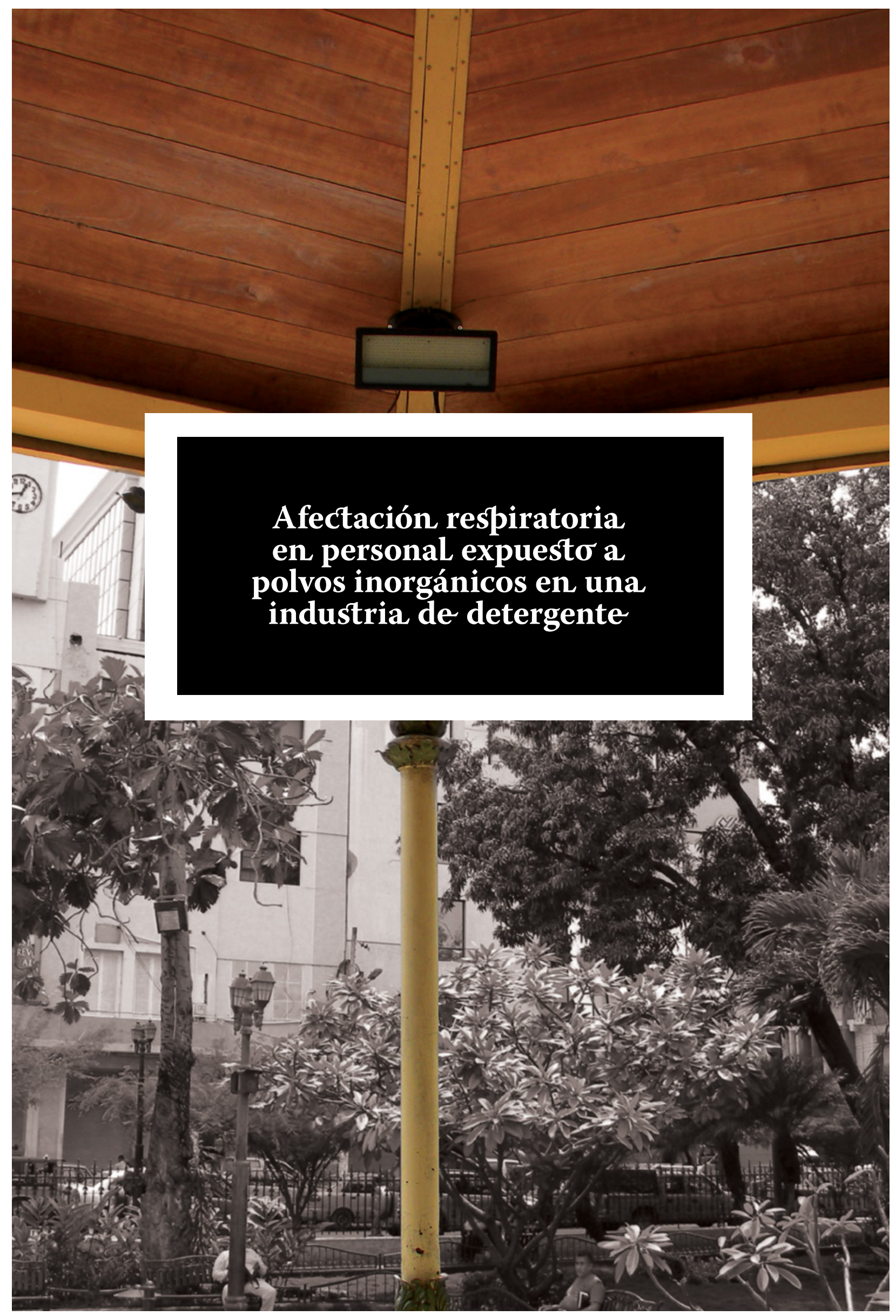




\title{
Afectación Respiratoria en Personal expuesto a polvos inorgánicos EN UNA INDUSTRIA DE DETERGENTE
}

\section{RESPIRATORY AFFECTATION IN PERSONNEL EXPOSED TO DUST IN A DETERGENT INDUSTRY}

\begin{abstract}
RESUMEN
La exposición a polvos inorgánicos diariamente en el lugar de trabajo, puede dar como resultado diversas enfermedades entre ellas las del sistema respiratorio, el objetivo de esta investigación es establecer la relación entre la exposición al polvo de detergente y la ocurrencia de afectaciones respiratorias en trabajadores expuestos, para ello se realizó un estudio descriptivo transversal en el que se analizaron los resultados de espirometría, ausentismo y antigüedad laboral de 158 trabajadores expuestos a polvo en una empresa de detergente en la Provincia de Manabí en el año 2018, los resultados demuestran el predominio en las características demográficas; género masculino con el 99,2\%, grupo etario de 29 a 39 años y una exposición laboral a polvos inorgánicos por elaboración de detergente del 33,3\% entre 3 y 4 años en el puesto, un pequeño porcentaje (12\%) presentó como afectación Infección Respiratoria Aguda (IRA) con reposo mayor a 48 horas hasta 96 horas y un 5 \% de los trabajadores presentaron una obstrucción en el resultado de la espirometría. Se concluye que hay una relación entre la exposición laboral a polvos inorgánicos y afectaciones respiratorias; a mayor tiempo de exposición mayor afectación pulmonar.
\end{abstract}

PALABRAS CLAVE: Detergente; enfermedades respiratorias; exposición; polvos inorgánicos; trabajadores.

Copyright (c) Revista San Gregorio 2019. ISSN: 1390-7247; eISSN: 2528-7907 @

\section{ABSTRACT}

Exposure to inorganic dust daily in the workplace, can result in various diseases including respiratory system, the purpose of this research is to establish the relationship between exposure to detergent powder and the occurrence of respiratory impairment in exposed workers, for this a cross-sectional descriptive study was carried out in which the results of spirometry, absenteeism and work seniority of 158 workers exposed to dust in a detergent company in the Province of Manabí in 2018 were analyzed, the results show the predominance in the demographic characteristics; male gender with $99.2 \%$, age group of 29 to 39 years and occupational exposure to inorganic dust by $33.3 \%$ detergent elaboration between 3 and 4 years in the position, a small percentage (12\%) presented as Acute Respiratory Infection (ARI) with rest greater than 48 hours up to 96 hours and $5 \%$ of the workers presented an obstruction in the result of the spirometry. It is concluded that there is a relationship between occupational exposure to inorganic dust and respiratory effects; the longer the exposure, the greater the lung involvement.

KEYWORDS: Detergent; respiratory diseases; exposure; inorganic dust; workers

Copyright (c) Revista San Gregorio 2019. ISSN: 1390-7247; eISSN: 2528-7907 @

Gema Evaldina Alcivar Tejena

1 Graduada de Maestría de Seguridad y Salud Ocupacional "Segunda

Graduada de Maestría de Seguridad y Salud Ocupacional "Segunda
Cohorte" de la Universidad San Gregorio de Portoviejo. Manabi. Ecuador

$\sim$

gemalcivar@hotmail.com

ARTÍCULO RECIBIDO: 30 DE ABRIL DE 2019

ARTÍCULO ACEPTADO PARA PUBLICACIÓN: 7 DE JUNIO DE 2019

ARTÍCULO PUBLICADO: 30 DE JUNIO DE 2019 


\section{INTRODUCCIÓN}

En el mundo se estima que la contribución del polvo, el humo y los gases a la enfermedad pulmonar obstructiva crónica (EPOC), en el puesto de trabajo, es del $15 \%$ de origen laboral (Vega y Rodríguez, 2015).

El material particulado es uno de los contaminantes atmosféricos más estudiados en el mundo, este se define como el conjunto de partículas sólidas y/o líquidas (a excepción del agua pura) presentes en suspensión en la atmósfera, que se originan a partir de una gran variedad de fuentes naturales o antropogénicas y poseen un amplio rango de propiedades morfológicas, físicas, químicas y termodinámica (Arciniégas, 2012, p. 195).

Expresan (Gómez, Henao, Molina \& Molina 2003) que:

El material particulado solo, o en combinación con otros contaminantes, especialmente dióxido de azufre (SO2), representa un grave riesgo para la salud, ya que las partículas menores de $10 \mu \mathrm{m}$ penetran directamente por las vías respiratorias, y ocasionan alteraciones en este sistema en diferentes sitios (p. 26).

El crecimiento poblacional tiene como resultado mayor consumo de productos de la línea diaria de higiene y cuidado personal. Según estudio realizado en el 2014 por Advance (2014) menciona que la mayor cantidad de hogares (95\%) en el país adquiere detergente en polvo con un consumo por familia al año de $24 \mathrm{~kg}$, lo que implica que haya cada vez más empresas manufactureras dedicadas a la producción de detergente y personal expuesto a diario a poluciones de polvo respirable en su ambiente de trabajo, los cuales podrían ser susceptibles a padecer enfermedades respiratorias.

Martínez y Rego (2000) mencionan que: “En el Reino Unido se observó que el 7\% de las consultas de atención primaria eran debidas a problemas relacionados con el trabajo y de ellas, el 10\% correspondían a síntomas respiratorios" (p. 631). En Ecuador no se tienen cifras exactas de atenciones médicas por problemas relacionados con el trabajo; además de que existe un subregistro de las posibles enfermedades ocupacionales que no son declaradas a la autoridad competente que en nuestro país la entidad encargada es Riesgos del Trabajo. Además, se ha observado que no existe diferencia en el cuadro clínico de las enfermedades laborales y no laborales, la diferencia está en el contexto y los antecedentes del paciente (Salinas \& Del Solar, 2015) esto puede generar un subregistro de las enfermedades ocupacionales. La (OIT, 2009) estima que anualmente ocurren 160 millones de casos de enfermedades relacionadas con el trabajo, existiendo un subregistro. A su vez el registro de las enfermedades y accidentes de trabajo se encuentra afectado por problemas en los sistemas de informaciones nacionales.

La Organización Mundial de la Salud (OMS) indica que "en América Latina y el Caribe, la notificación de enfermedades ocupacionales apenas alcanza entre el $1 \%$ y el $5 \%$ de los casos, ya que, por lo general, se registran solo aquellos que causan incapacidad sujeta a indemnización" (Álvarez, Castro, Ronquillo \& Rodríguez, 2011, p. 60).

Las enfermedades respiratorias en trabajadores expuestos al polvo lateritico fueron estudiadas por Vega y Rodríguez (2015) exposición a las enfermedades respiratorias en trabajadores expuestos al polvo lateritico en el cual mencionan que consideran que: “...la inhalación sostenida de polvos inorgánicos en el ambiente laboral puede originar diversas enfermedades respiratorias, conocidas como enfermedades pulmonares de origen ocupacional (EROC), las cuales representan una causa importante de mortalidad y discapacidad". (p. 337).

Para (Salinas y Del Solar, 2015) "El asma ocupacional es la enfermedad laboral respiratoria más frecuente en el mundo desarrollado. Diversos estudios estiman que entre el $10 \mathrm{a}$ $20 \%$ de los pacientes adultos con asma son de causa ocupacional, siendo esta condición subdiagnosticada" (p. 360).

Según registro que mantiene la Superintendencia de Compañías de nuestro país se 
presentan 64 Empresas Manufactureras dedicadas a la fabricación y distribución de jabones y detergentes, preparados para limpiar y pulir, perfumes y preparados de tocador; de ellas 5 industrias exportan estos productos a diferentes países con un $0.6 \%$ del total de las exportaciones durante el año 2017 (Superintendencia de Compañias Valores y Seguros, 2019).

Se toma como punto de estudio para la presente investigación una de estas Industrias Manufactureras exportadora ubicada en la provincia de Manabí, Cantón Manta con Clasificación Industrial Internacional Uniforme (CIIU) clase 2023 en la categoría Fabricación de jabones y detergentes, preparados para limpiar y pulir, perfumes y preparados de tocador (Superintendencia de Compañias Valores y Seguros, 2019) la misma que produce anualmente alrededor de 19.806,5 toneladas de detergente en polvo.

Con los antecedentes expuestos, el presente trabajo pretende establecer la relación entre la exposición al polvo de detergente y la ocurrencia de afectaciones respiratorias en trabajadores expuestos en una industria elaboradora de detergente de la Provincia de Manabí en el año 2018, de manera que permita identificar afectaciones respiratorias relacionadas con la exposición y la correlación del tiempo de exposición y funcionalidad pulmonar del personal expuesto a polvo de detergente.

\section{METODOLOGÍA}

El presente estudio fue de carácter observacional, descriptivo transversal, fueron evaluadas 158 pruebas de función pulmonar (espirometría) a trabajadores expuestos a polvos inorgánicos en una industria de detergente en la provincia de Manabí en el año 2018. El universo estuvo conformado por todos los trabajadores del Proceso de producción de detergente, el mismo que corresponde a 158 personas laboralmente activas; 157 masculinos y 1 persona de género femenino, esto puede deberse a que las actividades de la industria involucran tareas un poco más fuertes de realizar, con un rango de edad de 18 a 55 años y con exposición directa al polvo respirable por elaboración de detergente durante toda su jornada laboral.

Entre los criterios de exclusión que fueron considerados están las personas con antece- dentes de patologías respiratorias previas a su ingreso al proceso de elaboración de detergente, personas que realicen actividades relacionadas con polvos fuera de su jornada laboral en la empresa, la muestra final fue de 132 trabajadores, después de aplicado los criterios de exclusión.

Se revisó el ausentismo con referencia especial al asma, Infección Respiratoria Aguda (IRA), rinitis y dermatitis, se analizó la espirometría como prueba de función respiratoria con los criterios de aceptabilidad y repetibilidad de las mediciones y control de calidad de acuerdo al criterio NIOSH (A y B); el equipo utilizado para la toma de pruebas espirométricas fue el espirómetro Discovery II de la marca Futuremed, el mismo, fue calibrado diariamente con una jeringuilla de 3 litros de aire durante todo el año en que fueron tomadas las pruebas de espirometría, además cuenta con resultados automáticos de los criterios de calidad de la espirometría según los criterios NIOSH (A,B,C,D) y diagnostico a partir de los análisis estadísticos del equipo (normal, restrictiva, obstructiva y mixto) y una radiografía de tórax, así mismo se realizó una entrevista médica a todos los trabajadores incluidos en la muestra para analizar las variables confusoras como la exposición a polvo ambiental en su domicilio y trabajos relacionados con polvos fuera de su jornada laboral además, para conocer datos demográficos, ocupacionales y antecedentes de tabaquismo con las variables independientes de: edad, género, antigüedad laboral y la variable dependiente: presencia de síntomas respiratorios, dándole prioridad a la Infección respiratoria Aguda (IRA), que se define como variable cualitativa, nominal de 3 categorías (IRA sin reposo; Ausentismo laboral por IRA de más de 48 horas y ausentismo laboral por IRA de menos de 48 horas). La información fue recogida de la historia clínica ocupacional de los trabajadores y del Informe anual de morbilidad y ausentismo del Departamento Médico de la empresa. Se realizaron visitas al sitio de investigación y se utilizó la observación directa.

Se plantearon las siguientes hipótesis:

H1: La exposición a polvos inorgánicos repercute en la afectación respiratoria de los trabajadores de la empresa elaboradora de detergentes. 


\section{Hipótesis Nula}

H0: La exposición a polvos inorgánicos no repercute en la afectación respiratoria de los trabajadores de la empresa elaboradora de detergentes.

Para la demostración de la Hipótesis se utilizó la prueba de la correlación de Pearson, con un nivel de confianza del $95 \%$ y un nivel de significancia del $5 \%$.

Los resultados fueron tabulados en el programa estadístico IBM SPSS STATISTICS (Versión 25). El análisis que se llevó a cabo consistió en calcular estadísticos descriptivos (frecuencias) de las variables demográficas, ocupacionales y antecedentes tabáquicos; medidas de tendencia central (mediana, media y moda) y medidas de dispersión (mínimo y máximo), para el cruce de variables de tiempo de exposición y afectaciones se utilizó la correlación de Pearson.

La información científica y aporte para la presente investigación se realizó en las bases de datos de Pubmed, BMJ Journals, Medline, Elsevier, Dialnet, con el Google Académico, para la búsqueda de artículos científicos, revisiones sistemáticas, tesis de posgrado y doctorados; la misma que estuvo enfocada en investigaciones publicadas tanto en inglés como en español.

\section{ASPECTOS ÉTICOS Y LEGALES}

La investigación se ha realizado con los principios rectores, requisitos y buenas prácticas en la investigación mencionadas en las normas de bioética establecidas por Borroto Cruz (2015) como son el análisis de contenido, principio de respeto, principio de autonomía, validez de la información y el consentimiento informado a cada uno de los trabajadores que participan en el estudio, los mismos que fueron considerados como la base principal para la realización de la investigación además se ajustó a las consideraciones éticas establecidas en la Declaración de Helsinki (Manzini, 2000) así mismo se contó con la aprobación por parte del Departamento Legal de la Empresa de Detergente.

\section{MARCO LEGAL}

En la Constitución del Ecuador (2008) Art. 325, 326 y en el Decreto Ejecutivo 2393 (1986) se menciona la obligatoriedad de velar por la salud de los trabajadores en general, el con- venio de la Organización Internacional del Trabajo (OIT) adoptado por el Ecuador, C148Convenio sobre el medio ambiente de trabajo (contaminación del aire, ruido y vibraciones) de 1977. Existen Normas Técnicas Ecuatorianas como la NTE INEN 2348 (2013) y la NTE INEN 181 (2014) las mismas, estandarizan y regulan los tipos de protección respiratoria para material particulado.

A nivel internacional existen normas como la Administración de Seguridad y Salud Ocupacional (OSHA, 2019) y la Conferencia Americana de Higienistas Industriales Gubernamentales (ACGIH, 2019) las cuales son específicas para áreas de trabajo cuyo objetivo es evaluar cuantitativamente los riesgos a los que están expuestos los trabajadores. En Ecuador no existen leyes, reglamentos y normas específicos para Partículas Inhalables y Partículas Respirables en ambientes laborales, es por ello que se toman como referencia las internacionales como las OSHA y las ACGIH.

\section{RESULTADOS}

A partir de la tabulación de las preguntas de la entrevista médica al grupo de trabajadores del proceso de elaboración de detergentes, dentro de las edades comprendidas entre 18 y 55 años el grupo de mayor población es el de 29 a 39 años con un $43,2 \%$.

La antigüedad laboral de los trabajadores en estudio fue variada; un 33,3\% de ellos con mayor predominio se encuentran en el grupo de 3 a 4 años en el puesto de trabajo y en menor predominio un $15 \%$ se encuentran en el grupo de 8 años y más de labores en el proceso de detergentes

El $94,7 \%$ de los trabajadores no tienen antecedentes de tabaquismo sin embargo un $2 \%$ manifestó haber fumado antes sin ser dependientes a la nicotina y un $3 \%$ de ellos de acuerdo al test de fargestrom son poco dependientes a la nicotina con un consumo entre 1 y 3 cigarrillos al día.

El 97,7\% de los trabajadores en estudio refirió que no ha habitado en lugares donde exista presencia de polvos, mientras que un 2,3\% de ellos si menciona haber residido en lugares con exposición a polvo ambiental como polvos industriales y el suspendido en el aire por el paso de vehículos en las calles. 
Los resultados de las mediciones de concentración de polvo respirable dentro del proceso de elaboración de detergentes del año 2016 en el área de detergentes son superiores para la norma ACGIH que es de $3 \mathrm{mg} / \mathrm{m} 3$ y supera la norma OSHA de $5 \mathrm{mg} / \mathrm{m} 3$.

El 100\% de la muestra mencionó no tener antecedentes respiratorios antes de entrar a laborar en el proceso de detergentes, así mismo refieren usar equipo de protección respiratoria completa mientras están dentro del proceso y que ninguno ejecuta actividades relacionadas con polvo luego de su jornada laboral en la industria, se observó que el 98,5\% de los trabajadores mencionan no haber sido diagnosticados con ninguna patología, mientras que un $0,8 \%$ refiere haber sido diagnosticado con Diabetes y un $0,8 \%$ con Hipertensión Arterial.

El 59,1\% de la población en estudio no presentó afectaciones respiratorias durante el tiempo de exposición laboral en el proceso de detergente, un $23,5 \%$ presentó un reposo menor a 48 horas con diagnóstico de IRA y un $12,1 \%$ ameritó reposo con diagnóstico de IRA mayor a 48 horas. Mientras que el $88,6 \%$ de la población no presentó otras afectaciones a la salud.

Del personal en estudio se obtuvo una espirometría normal bajo los criterios NIOSH A y B (calidad de la espirometría) durante el tiempo de exposición laboral en el proceso de detergente; el 5,3 \% tuvo una espirometría restrictiva;

En la tabla $\mathrm{N}^{\circ} 1$ (Ver en Anexos) se evidencia que, en relación al tiempo de exposición de los trabajadores en el rango de 0 a 2 años de exposición 1 persona presenta espirometría restrictiva, en los demás rangos comprendidos entre 3 a 4 años, 5 a 7 años, y 8 años en adelante, 2 personas presentan restricción en su espirometría en cada uno de los rangos citados.

En la tabla $N^{\circ} 2$ (Ver en Anexos) se puede observar que, en relación al tiempo de exposición de los trabajadores y la presencia de afectaciones por IRA, en el rango de 0 a 2 años se encuentran afectaciones por IRA sin ameritar reposo médico, el rango comprendido entre 3 a 4 años, 5 a 7 años, y 8 años en adelante se presentan afectaciones por IRA con reposos de hasta más de 48 horas en cada uno de los rangos citados.

Se pudo apreciar que a mayor edad y exposición laboral se presentan afectaciones respiratorias como es la IRA con reposo de hasta 96 horas; así mismo sucede con el Diagnostico de Rinitis sin reposo con un 3\% del total de los trabajadores. En ambos casos, IRA y Rinitis se empiezan a presentar después de los 3 años de labores y estas se mantienen aún a partir de los 8 años de exposición laboral.

Se comprueba la hipótesis del investigador mediante la correlación de Pearson dado que el p- valor es menor a 0.05 , por consiguiente, existe suficiente evidencia estadística para afirmar que la exposición a polvos inorgánicos dentro del ambiente laboral incide en la presencia de afectaciones respiratorias.

Nivel de significancia $=5 \%=0.05$

A través de su índice $\mathrm{R}$ y Rho de Pearson se hace la medida de correlación, lo cual indica una relación positiva de nivel escasa relación.

\section{DISCUSIÓN}

A nivel mundial se han realizado muchos estudios por exposición laboral a polvos inorgánicos; Martinez, Quero, Isidro y Rego (2001); Vega y Rodriguez (2015); Callahan et al. (2019); Arciniégas (2012); Gómez et al. (2003) y muchos más hacen referencia a las enfermedades respiratorias ocupacionales resultantes de la exposición a material particulado por polvos.

Asas (2018); Sánchez (2017); Vilema (2018) y Jiménez (2014) han realizado en nuestro país estudios de material particulado y su relación con afectaciones a la salud por exposición a polvos en Industrias de automotores, calzados, harinas y polvo por elaboración de billetes del Banco Central del Ecuador respectivamente.

En el Ecuador no se han realizado investigaciones sobre la relación de material particulado por polvo de detergente y enfermedades pulmonares; sin embargo, Gina Sanclemente (2016) realizó un "Diseño de un programa de vigilancia de salud para disminuir los efectos de reacción alérgica en la población expuesta a enzimas blanqueadoras que trabajan dentro 
de una fábrica de detergentes en Guayaquil" (p. 04).

Francisco Segarra como se citó en (Vega \& Rodriguez 2015 p. 344) menciona que "el tiempo de exposición necesario para desencadenar la enfermedad es muy variable: depende de la naturaleza de las partículas inhaladas, intensidad de la exposición, entre otros". Asi mismo, Cullinan, Tarlo y Nemery (2003) en su estudio de la prevención del asma ocupacional mencionan que: "el sexo y edad son variables independientes, pero a más edad, el pronóstico es peor" (p. 854).

Se calcula que la superficie de los alvéolos pulmonares alcanza unos $70 \mathrm{~m} 2$, y es ventilada por unos 10.000 litros de aire diarios, por lo que el pulmón resulta un órgano muy accesible a la inhalación de una suspensión de partículas sólidas en el aire, que denominamos polvo. (Martínez et al. 2001 (p.61). Se concuerda con lo señalado por Sánchez (2005) quien dice que la antigüedad laboral, es un papel fundamental para la detección de enfermedades ocupacionales por considerarse el tiempo que un individuo está expuesto a una sustancia química, efectos adversos directamente relacionados con la exposición, a mayor tiempo de exposición, mayor posibilidad de desarrollar algún efecto adverso a la salud.

Los componentes con especial importancia a las enzimas utilizadas en la elaboración de detergentes son sustancias inhalables, irritativas o alergénicas que pueden causar afectaciones sobre la salud de los trabajadores inmersos en el proceso de industrialización.

Por consenso de expertos, dada la poca evidencia que existe, se indica que los trabajadores con Asma Ocupacional (AO) derivada de la exposición a agentes irritantes, pueden seguir expuestos siempre que se tomen las medidas ingenieriles que eviten un episodio accidental agudo o nuevo en la enfermedad y se usen Equipos de Protección Personal Respiratorios (EPP). Se debe mantener el tratamiento medicamentoso e implementar las prestaciones económicas de incapacidad permanente (Dykewicz, 2009).

Manifiesta Gómez et al. (2003) que: "aun a bajas concentraciones $(20 \mu \mathrm{g} / \mathrm{m} 3)$ la contaminación por partículas suspendidas totales (PST) puede incrementar el riesgo de las infecciones respiratorias agudas (IRA), los epi- sodios asmáticos y de otras enfermedades respiratorias". (p. 27).

De los trabajadores estudiados en la presente investigación se aprecia un pequeño porcentaje de trabajadores con afectaciones de Infección Respiratoria Aguda (IRA) durante todo el tiempo de exposición laboral, la cual ameritó un reposo mayor a 48 horas y menor a 96 horas, estos trabajadores tenían una exposición laboral de 3 a 4 años, sin poder establecer que sea relacionado al trabajo por la poca duración de la enfermedad y ausencia de otros síntomas respiratorios.

Para autores como García et al. (2013) expresan que "La espirometría es una prueba básica para el estudio de la función pulmonar, y su realización es necesaria para la evaluación y el seguimiento de las enfermedades respiratorias". (p. 369).

Para que una prueba de espirometría nos pueda dar un resultado correcto una de las variantes a verificar es la calidad, la misma que está dada por grados de calidad siendo las A y B de buena calidad por considerarse que cumplen los criterios de calidad de la curva y repetitibilidad en las 3 pruebas básicas, las espirometrías de grado $C$ dan una calidad suficiente y las de los grados D y superiores no son válidas para interpretación. García et al. (2013)

Por lo anteriormente evidenciado en los resultados estadísticos de esta investigación llama la atención un pequeño número de trabajadores que presentan alteración de los patrones funcionales respiratorios encontrándose en los grupos de 3 años en adelante de exposición laboral, y un solo caso se evidenció en el grupo de exposición comprendido entre 0- 2 años.

Los resultados de las espirometrías de los trabajadores fueron realizados con especificaciones NIOSH de buena calidad A y B, se encontró el 5\% de ellas con un patrón restrictivo.

Las principales variables de la espirometría forzada son la capacidad vital forzada (FVC) y el volumen espiratorio forzado en el primer segundo (FEV1). El FEV1 corresponde al volumen máximo de aire exhalado en el primer segundo de la maniobra de FVC, también expresado en litros. García et al. (2013, p. 402). 
En un estudio realizado en Inglaterra en el 2012 por Fishwick et al. (2012, p. 279) mencionan que: "el riesgo del desarrollo de asma ocupacional es mayor después del inicio de rinitis ocupacional".

En la investigación realizada se presentaron en menor frecuencia otras afecciones a la salud tales como dermatitis, micosis y rinitis; todas ellas de corta duración sin ser clasificadas como patológicas, pero si como reacción a la exposición de enzimas elaboradoras de detergente. Esto puede estar en función a las bajas concentraciones de polvo ambiental dentro del proceso de elaboración de detergentes, la aplicación constante de las medidas de seguridad y el correcto uso del equipo de protección personal durante su jornada laboral.

\section{CONCLUSIONES}

A mayor tiempo de exposición mayor afectación pulmonar. Se comprueba que la exposición a polvos inorgánicos producto de un proceso laboral como es la elaboración de detergente en polvos incide en la presencia de afectaciones respiratorias.

De lo anteriormente expuesto es importante mencionar que el riesgo de afecciones respiratorias se relaciona con la magnitud y exposición laboral y que el componente restrictivo de las alteraciones de la función pulmonar comienza en las pequeñas vías aéreas, pero que también se puede evitar gracias a los mecanismos de defensa y protección del trabajador.

La población que labora en esta empresa a pesar de estar expuesta no está afectada en su mayoría, esto debido a que todos usan sus equipos de protección respiratoria, no tienen antecedentes tabáquicos importantes y no viven en domicilios donde exista mayor contaminación de polvo.

Como está documentada en la literatura y se ha estudiado en esta investigación la exposición a enzimas de detergente, presenta aparte de problemas a nivel del sistema respiratorio, problemas a nivel de la piel, como son la dermatitis leve en cualquier momento de su exposición laboral.

Las pruebas radiográficas reportan el 100\% de normalidad lo que coincide con la ausen- cia de síntomas respiratorios y las pruebas de función pulmonar.

\section{RECOMENDACIONES}

La vigilancia de la salud de los trabajadores expuestos a polvos inorgánicos suspendidos en el lugar de trabajo es imprescindible para evitar la generación de enfermedades ocupacionales por lo que se recomienda:

1. Seguir con la vigilancia médica ocupacional y ambiental.

2. Continuar con el uso adecuado de protección personal respiratoria.

3. Socializar y sensibilizar sobre el riesgo del material particulado por polvo.

4. Control de las horas laborables dentro de la jornada.

5. Mediciones de polvo respirable, caracterización del material particulado.

6. Seguimiento de los trabajadores con signos y síntomas de enfermedades respiratorias. 


\section{REFERENCIAS BIBLIOGRÁFICAS}

Administración de Seguridad y Salud Ocupacional. (03 de 02 de 2019). Departamento de Trabajo de Los Estados Unidos. Obtenido de https://www.osha.gov

Advance. (2014). Línea Base Aceites y Detergentes. Recuperado el 19 de 01 de 2019, de http://www. quitoambiente.gob.ec

Castelló., M Castro Almarales, R L. \& Díaz, R. (2011). Asma ocupacional, una aproximación necesaria al problema. Revista cubana de Salud y Trabajo, 59-65.

Arciniégas Suárez, C. A. (enero-junio de 2012). Diagnóstico y control de material particulado: Partículas suspendidas totales y fracción respirable PM 10. Luna Azul, 195-213. Recuperado el 05 de Marzo de 2019, de https://www.redalyc.org

Asamblea Constituyente. (29 de octubre de 2008). Constitución de la República del Ecuador. Quito, Ecuador: Registro Oficial 449

Asas Toapanta, J. F. (2018). La exposición a material particulado y su repercusión en la salud de los trabajadores en el sector industrial carrocero. Tesis de Maestría, Universidad Técnica de Ambato, Facultad de Ingeniería en Sistemas, Electrónica e Industrial, Maestría en Seguridad e Higiene Industrial y Ambiental.

Borroto Cruz, E. (2015). Bioética e investigación: Puente hasta el presente y para el futuro. Revista San Gregorio, 6-15.

Callahan, C. L., Freisen, M. C., Locke, S. J., Dopart, P. J., Stewart, P. A., Schwart, K.,... Purdue, M. P. (13 de 02 de 2019). Investigación de casos y controles de exposición ocupacional al plomo y cáncer de riñón. Medicina Ocupacional y Ambiental. Obtenido de https://oem.bmj. com

Conferencia Americana de Higienistas Industriales Gubernamentales. (03 de 02 de 2019). Conferencia Americana de Higienistas Industriales Gubernamentales. Obtenido de https://www.acgih.org

Cullinan, P., Tarlo, S., \& Nemery, B. (2003). The prevention of occupational asthma. European Respiratoy Journal, 22(5), 853-860.

Dykewicz, M. S. (2009). Occupational asthma: current concepts in pathogenesis, diagnosis, and management. Journal of Allergy and Clinical Immunology, 123(3), 519528.

Fishwick, D., Barber, C. M., Bradshaw, L. M., Barraclough, R., Burge, S., \& Corne, J. (2012). Standards of care for occupational asthma. Chest Clinic, 3(67), 278-280.

García, F., Calle, M., Burgos, F., Del Campo, F., Casan, P., Galdizf, J., . . . Puente, L. (2013). Espirometria. Archivos de Bronconeumologia, 369-412.

Gómez, A., Henao, E., Molina, E., \& Molina, F. (2003). Evaluación de las partículas suspendidas totales (PST) y partículas respirables (PM10) en la zona de Guayabal. Revista Facultad de Ingeniería Universidad de Antioquia, 24-33.
Instituto Ecuatoriano de Normalización. (05 de 2013) Seguridad. Respiradores de protección contra partículas suspendidas en el aire. Requisitos. NTE INEN (2384), 1.

Jiménez Ollague, D. F. (2014). Incidencia de rinitis alérgica con relación a la exposición de material particulado emitido por los billetes en el área de especies monetarias del Banco Central del Ecuador. Tesis de maestría, Universidad de Guayaquil, Facultad de Ingeniería Industrial. Maestría en Seguridad, Higiene Industrial y Salud Ocupacional.

Laborales, M. D. (1986). Reglamento de Seguridad y Salud de los Trabajadores y Mejoramiento del Medio Ambiente de Trabajo. Decreto Ejecutivo (2393).

Manzini, J. L. (2000). Declaración de Helsinki: principios éticos para la investigación médica sobre sujetos humanos. Acta bioethica, 6(2), 321-334.

Martínez González, C., \& Rego Fernández, G. (2000) Enfermedades respiratorias de origen ocupacional. Archivos de Bronconeumologia, 631-644. Obtenido de https://www.sciencedirect.com

Martínez, C., Quero, A., Isidro, I., \& Rego, G. (2001) Enfermedades pulmonares profesionales por Inhalación de polvos inorgánicos. : MED HUM,

OIT. (2009). Organización Mundial del Trabajo. Obtenido de https://www.ilo.org

Organización Internacional del Trabajo. (junio de 1977). Recuperado el 20 de 02 de 2019, de https://www.ilo.org/ dyn/normlex/

Salinas F, M., \& Del Solar, J. A. (05 de 2015). ENFERMEDADES RESPIRATORIAS OCUPACIONALES. Revista Médica Clínica Las Condes ELSEVIER, 26(3), 357-366.

Sánchez Rosero, C. H. (2017). Material particulado y su incidencia en la salud de los trabajadores en la Empresa de Calzado CM Original. Tesis de Maestría, Universidad Técnica de Ambato, Facultad de Ingeniería en Sistemas, Electrónica e Industrial. Maestría en Seguridad e Higiene Industrial y Ambiental.

Sánchez, M. M. (2005). Efectos neuroconductuales en trabajadores expuestos a solventes orgánicos en la industria petrolera. Tesis Doctoral, Universidad Del Zulia, Maracaibo, Venezuela. Obtenido de http://tesis. luz.edu.ve

Sanclemente Lainez, G. J. (2016). Diseño de un programa de vigilancia de salud para disminuir los efectos de reacción alérgica en la población expuesta a enzimas blanqueadora que trabajan dentro de una fábrica de detergentes. Universidad de Guayaquil, Facultad de Ingeniería Industrial. Maestría en Seguridad, Higiene Industrial y Salud Ocupacional. 
ANEXOS

\section{Espirometría y Tiempo de Exposición laboral}

\begin{tabular}{|c|c|c|c|c|c|c|}
\hline & & \multicolumn{4}{|c|}{ Tiempo de Exposición laboral } & \multirow[b]{2}{*}{ Total } \\
\hline & & $\begin{array}{c}0-2 \\
\text { años }\end{array}$ & $\begin{array}{c}3-4 \\
\text { años }\end{array}$ & $\begin{array}{c}5-7 \\
\text { años } \\
\end{array}$ & $\begin{array}{c}8 \text { años en } \\
\text { adelante }\end{array}$ & \\
\hline \multirow[t]{3}{*}{ Normal } & $\%$ Tiempo de & 39 & 42 & 26 & 18 & 125 \\
\hline & Exposición laboral & & & & & \\
\hline & & $97,5 \%$ & $95,5 \%$ & $92,9 \%$ & $90,0 \%$ & $94,7 \%$ \\
\hline \multirow[t]{4}{*}{ Restrictiva } & $\%$ Tiempo de & 1 & 2 & 2 & 2 & 7 \\
\hline & Exposición laboral & & & & & \\
\hline & & $2,5 \%$ & $4,5 \%$ & $7,1 \%$ & $10,0 \%$ & $5,3 \%$ \\
\hline & $\%$ Tiempo de & 40 & 44 & 28 & 20 & 132 \\
\hline Total & Exposición laboral & & & & & \\
\hline
\end{tabular}

Tabla 1. Tiempo de exposición laboral y resultado de espirometría

Fuente: Historia Clínica Ocupacional

\begin{tabular}{|c|c|c|c|c|}
\hline \multicolumn{2}{|c|}{ Tiempo de Exposición laboral } & \multicolumn{2}{|c|}{ Frecuencia Porcentaje } & \multirow{2}{*}{$\begin{array}{l}\text { Porcentaje } \\
\text { acumulado }\end{array}$} \\
\hline 2 años & Ninguna & 35 & 26,52 & \\
\hline & Ira con reposo $<48 \mathrm{~h}$ & 5 & 3,79 & 30,31 \\
\hline \multirow[t]{4}{*}{ 3-4 años } & Ninguna & 21 & 15,91 & 46,22 \\
\hline & IRA sin reposo & 1 & 0,76 & 46,98 \\
\hline & Ira con reposo $<48 \mathrm{~h}$ & 15 & 11,36 & 58,34 \\
\hline & Ira con reposo $>48 \mathrm{~h}$ & 7 & 5,30 & 63,64 \\
\hline \multirow[t]{4}{*}{ 5-7 años } & Ninguna & 15 & 11,36 & 75 \\
\hline & IRA sin reposo & 2 & 1,52 & 76,52 \\
\hline & Ira con reposo $<48 \mathrm{~h}$ & 6 & 4,55 & 81,07 \\
\hline & Ira con reposo $>48 \mathrm{~h}$ & 5 & 3,79 & 84,86 \\
\hline 8 años en & Ninguna & 7 & 5,30 & 90,16 \\
\hline \multirow[t]{4}{*}{ adelante } & IRA sin reposo & 4 & 3,03 & 93,19 \\
\hline & Ira con reposo $<48 \mathrm{~h}$ & 5 & 3,79 & 96.98 \\
\hline & Ira con reposo $>48 \mathrm{~h}$ & 4 & 3,02 & 100,0 \\
\hline & Total & 132 & 100,0 & \\
\hline
\end{tabular}

Tabla 2. Antigüedad laboral y afectaciones respiratorias.

Fuente: Historia Clínica Ocupacional 